\title{
miR-768-3p is involved in the proliferation, invasion and migration of non-small cell lung carcinomas
}

\author{
ZUOZHOU XIE, WEIQIANG CHEN, YINGHUA CHEN, XIANG WANG, WENYONG GAO and YI LIU
}

Department of Respiratory and Critical Medicine, No. 2 People's Hospital of Kunming, Kunming, Yunnan 650204, P.R. China

Received November 2, 2016; Accepted September 18, 2017

DOI: $10.3892 /$ ijo. 2017.4133

\begin{abstract}
Altered microRNA expression has been found to be a common feature of several cancers, including lung carcinomas. However, the possible roles of miR-768-3p in the pathological changes of lung carcinomas are still unknown. The aim of the present study was to investigate the expression and possible effects of miR-768-3p in human non-small cell lung carcinomas (NSCLC). Eighty-three NSCLC patients attending the clinic of Kunming Hospital were invited to participate in the study. Their tumor samples were obtained for qRT-PCR analysis. Human NSCLC cell lines, A549 and HCC4006, were employed and transfected with either miR-768-3p mimics or miR-768-3p antagomir. Following transfection, the in vitro and in vivo proliferation, apoptosis fractions, migration and invasion of NSCLC cells were evaluated. The data revealed that: i) upregulated miR-768-3p in tumors were associated with the clinicopathological features of NSCLC patients; ii) inhibiting miR-768-3p function by miR-768-3p antagomir induced distinctly apoptosis and Fas/FasL expressional alteration of NSCLC cells; iii) miR-768-3p antagomir transduction also decreased the viability, migration and invasion, as well as MMP-2 and MMP-9 activities in A549 and HCC4006 cells; and iv) miR-768-3p antagomir transfection also inhibited the growth and proliferation of NSCLC xenografts in nude mice. The present results suggested that abnormal elevated miR-768-3p in NSCLC tumors and cell lines played important roles in NSCLC carcinogenic progression, and the targeting of miR-768-3p might be a potential therapeutic strategy for the treatment of NSCLC.
\end{abstract}

\section{Introduction}

Lung cancer (LC) is the leading cause of cancer-related deaths worldwide (1), with 1.6 million new cases and 1.38 million deaths annually $(2,3)$. Lung cancer is a heterogeneous disease

Correspondence to: Professor Yi Liu, Department of Respiratory and Critical Medicine, No. 2 People's Hospital of Kunming, No. 42 Ciba North Road, Panlong, Kunming, Yunnan 650204, P.R. China E-mail: yiliu1592015@126.com

Key words: non-small cell lung carcinomas, miR-768-3p, proliferation, apoptosis, migration, invasion and a leading cause of cancer-related death in most developed countries. On the basis of differences in histology, LC is roughly divided into small- and non-small cell LC (SCLC and NSCLC, respectively). The latter, which accounts for approximately $85 \%$ of all LC malignancies and the overall 5-year survival of patients with NSCLC, remains approximately $15-20 \%(4,5)$.

MicroRNAs (miRNAs or miRs) are evolutionarily conserved non-coding single-stranded RNA molecules of approximately 18-25 nucleotides. They negatively regulate gene expression at the post-transcriptional level by either degrading the target mRNA or inhibiting translation of the mRNA into functional protein $(6,7)$. miRNAs have indeed been shown to regulate multiple hallmarks of cancer, for example, increased proliferation and evasion of cell death $(8,9)$. Recently, altered expression of miRNAs have been found to be a common feature of several cancers. An increasing number of reports have implicated a role for miRNAs in lung cancer progression $(10,11)$. miRNAs are potential targets for treating NSCLC carcinomas (12), and research has focused on the diagnostic and prognostic potential of different miRNAs in NSCLC. It is believed that miRNA expression is important in NSCLC development $(13,14)$. Functional elucidation of miR$768-3 p$ shed light on the specific roles in carcinogenic events of various cancers. However, the possible roles of miR-768-3p in pathogenic process and development involved in human NSCLC have not been illuminated previously.

The aim of the present study was to investigate the expression of miR-768-3p in human NSCLC and the possible effects of miR-768-3p alteration on the biological behavior of human NSCLC cells. Firstly, 83 patients attending the clinic of Kunming Hospital (pathologically diagnosed as NSCLC), between June 2010 and December 2011, were invited to participate in the study. Their surgical tumor samples were obtained for qRT-PCR analysis. Human NSCLC cell line, A549 and HCC4006, were employed and transfected with either miR-768-3p mimics or miR-768-3p-antagomir. Transfection efficiency was evaluated by qRT-PCR. The cell proliferation and apoptosis fractions were also evaluated by MTT assay and flow cytometry (FCM). The scratch wound assay and Transwell assays were also employed to explore the effects of miR-768-3p on the in vitro migration and invasion of NSCLC cells, respectively. We also used nude mice bearing NSCLC xenograft to evaluate the possible effects of miR-768-3p on NSCLC growth and proliferation in vivo. 


\section{Materials and methods}

Ethics approval and consent to participate. The study related to humans was approved by The Regional Committee for Research Ethics. The study complied with all the relevant national regulations, institutional policies in accordance with the tenets of the Helsinki Declaration.

Subject. The anonymized patients $(\mathrm{n}=83)$ diagnosed with NSCLC within the period from June 2010 to December 2011 at the Hospital (No. 2 People's Hospital of Kunming, Kunming, China), were employed in the present study. All NSCLC patients had primary lung cancer without any co-existing illness nor treatment, including drugs, chemotherapy and radio-chemotherapy. All participating patients voluntarily signed the informed consent. All cases were reviewed by three independent pathologists in our hospital and the clinical stages of NSCLC referenced to the criteria recently established by the National Comprehensive Cancer Network (NCCN). The TNM stages of tumors were determined according to the standard TNM classification system of the International Union Against Cancer (7th edition) (http://www.uicc.org/).

Sample preparation. For the present study, lung cancer and their adjacent normal tissues were collected after video-assisted fiberoptic bronchoscopy. The tissues were immediately stored at $-80^{\circ} \mathrm{C}$ until nucleic acids isolation.

Cell culture and treatment. The bronchoepithelial cell line BEAS-2B (human bronchial epithelium), A549 and HCC4006 human NSCLC cell line was purchased from the Cell Bank of the Chinese Academy of Sciences (Shanghai, China). The cell lines were cultured in Dulbecco's modified Eagle's medium (DMEM) with low glucose (GE Healthcare Life Sciences, Vienna, Austria), supplemented with $10 \%$ fetal calf serum (FCS; Sigma-Aldrich, Munich, Germany) and $100 \mathrm{U} / \mathrm{ml}$ penicillin, $100 \mu \mathrm{g} / \mathrm{ml}$ streptomycin and $2 \mathrm{mM}$ Lglutamine (PAA Laboratories; GE Healthcare Life Sciences). The cells were cultivated in cell culture flasks (Falcon ${ }^{\circledR}$; Becton-Dickinson Austria $\mathrm{GmbH}$, Schwechat, Austria) at $37^{\circ} \mathrm{C}$ in an atmosphere of $5 \% \mathrm{CO}_{2}$ until the desired cell confluence for further experiments was reached (15).

Upregulation or downregulation of miR-768-3p expression in cell lines was achieved by transduction with miR-768-3p-mimic (at a final concentration of $20 \mathrm{pmol} / \mu \mathrm{l}$ ) or miR-768-3p-antagomir (at a final concentration of $25 \mathrm{pmol} / \mu \mathrm{l}$ ) by using Lipofectamine ${ }^{\mathrm{TM}} 2000$ system (Invitrogen), respectively. The miR-768-3p-mimic, miR-768-3p-antagomir and their matched miR-NC were obtained from Guangzhou RiboBio Co., Ltd. (miR-Rib ${ }^{\mathrm{TM}}$ miRNA; Guangzhou, China). These cell lines treated with different reagents seeded in 6-well plates were harvested for isolated RNAs $48 \mathrm{~h}$ after transfection.

Quantitative RT-PCR. Total RNA from serum and tissue samples was prepared using a TRIzol and miRNeasy Mini kit (Qiagen) according to the manufacturer's instruction.

Reverse transcription and real-time PCR (RT-PCR) quantification of miRNA. cDNA was synthesized from total
RNA using gene-specific primers according to the TaqMan MicroRNA assay as per the protocol of the manufacturer (Applied Biosystems, Foster City, CA, USA). Quantitative PCR of miRNA was performed using an Applied Biosystems 7300 Sequence Detection system. The $10 \mu 1$ PCR reaction contained $0.67 \mu 1$ reverse transcription product, 1X TaqMan Universal PCR Master Mix, and $1 \mu \mathrm{l}$ of the primer and probe mix, according to the TaqMan MicroRNA assay protocol (Applied Biosystems) (16). The relative expression levels of genes were determined using the $2^{-\Delta \Delta \mathrm{Ct}}$ analysis method. The snU6 served as control.

MTT assay. Cell viability was determined using the tetrazolium salt 3-(4,5-dimethylthiazol-2-yl)-2,5-diphenyltetrazolium bromide (MTT) assay. Briefly, cells were plated into 96-well culture plates at an optimal density of $5 \times 10^{3}$ cells $/ \mathrm{ml}$ in $200 \mu \mathrm{l}$ of culture medium per well. After $24-96 \mathrm{~h}$ of culture, $20 \mu \mathrm{l}$ of $5 \mathrm{mg} / \mathrm{ml}$ MTT was added to each well and incubated at $37^{\circ} \mathrm{C}$ for $4 \mathrm{~h}$. The medium was then gently aspirated and $150 \mu \mathrm{l}$ of dimethyl sulfoxide (DMSO) was added to each well to solubilize the formazan crystals. The optical density of each sample was immediately measured using a microplate reader (Bio-Rad Laboratories, Hercules, CA, USA) at $490 \mathrm{~nm}$.

Apoptosis assay. A propidium iodide (PI) and Annexin V-FITCflow cytometry assay (BD Biosciences) was used to detect the apoptosis rate in the cells after various treatment transfection. Briefly, cells were harvested in complete RPMI-1640 medium and centrifuged at 1,000 rpm for $5 \mathrm{~min}$. Each of the cell lines was washed with $1 \mathrm{X}$ phosphate-buffered saline (PBS) and stained with $50 \mu \mathrm{g} / \mathrm{ml}$ PI and Annexin V-FITC, following the manufacturer's instructions.

Western blot analysis. The protein expression of apoptosisrelated proteins in NSCLC cells was determined by using western blot analysis according to the protocol described before. Briefly, cell samples were lysed on ice for $30 \mathrm{~min}$ in CytoBuster Protein Extraction Buffer (Novagen, Madison, WI, USA) and $50 \mu \mathrm{g}$ of protein was used for $10 \%$ sodium dodecyl sulfate polyacrylamide gel electrophoresis (SDS-PAGE). The protein was then transferred to a nitrocellulose (NC) membrane and was sealed with Tris-buffered saline Tween-20 (TBST) containing 5\% non-fat milk powder. The membrane was subsequently incubated with goat anti-human Fas (1:1,000; Cell Signaling Technology, Danvers, MA, USA), FasL (1:800; Santa Cruz Biotechnology, Santa Cruz, CA, USA) proteins, and rabbit anti-human GAPDH (1:1,000; R\&D Systems, Minneapolis, MN, USA) at $4^{\circ} \mathrm{C}$ overnight, respectively. After washing in TBST, the membrane was incubated with HPR conjugated secondary antibodies $(1: 1,000)$ at $25^{\circ} \mathrm{C}$, and the protein quantity was determined using electrochemiluminescence (ECL) technique (BestBio, Oakland, CA, USA). The results were photographed using the JS Gel Imaging System (Shanghai Peiqing Science and Technology Co., Ltd., Shanghai, China) and the gray density was calculated using SensiAnsys software (Shanghai Peiqing Science and Technology).

Migration assays. Scratch wound assay employed to detect the migration of the NSCLC cell lines before and after various reagent treatments, as described before (17). Briefly, the A549 
and HCC4006 cells transfected with miR-768-3p-mimics, miR-768-3p-antagomir and control miRNA-NC, at $\sim 80 \%$ confluency were seeded onto 6-well plates and incubated at $37^{\circ} \mathrm{C}$ for $24 \mathrm{~h}$. Then, a vertical scratch wound was made through the center of each well using a 10- $\mu$ l pipette tip. The cells were then washed three times with PBS to remove the scratched cells, and fresh serum-free medium was transferred. After $12 \mathrm{~h}$, the cells were examined by light microscopy at a magnification of $\times 200$ to determine the resealing of the cell monolayer.

Cell invasion assays. We employed BioCoat Matrigel invasion chambers (BD Biosciences, Bedford, MA, USA) to compare the effect of miR-768-3p overexpression or knockdown on in vitro invasion of NSCLC cells as previously described $(16,18)$. Briefly, for the invasion assay, Costar Transwell $8 \mu \mathrm{m}$ inserts were coated with $50 \mu \mathrm{g}$ reduced serum Matrigel (BD Biosciences). Invasion chambers were coated with Matrigel and $1 \times 10^{6}$ cells were added per chamber. Medium supplemented with $10 \%$ fetal bovine setum (FBS) was used in the lower chamber. Following incubation of cells that had invaded through the membrane that were fixed and stained with crystal violet before the membrane was removed and mounted on a slide for microscopic assessment. Invasive cells were visualized at $x 40$ magnification and the number of cells in five random fields was counted and an average calculated.

Matrix metalloprotein-2/9 (MMP-2/9) activity assay. The activity of migration associated matrix metalloproteines, MMP-2 and MMP-9, were determined by QuickZyme MMPs activity assay (QucikZyme BioSciences, Leiden, The Netherlands) according to the manufacturer's protocols. Briefly, after transfection, cells were washed with fresh medium and replaced with serum-free medium. After additional $24 \mathrm{~h}$, the medium was collected and centrifuged at $10,000 \mathrm{x}$ g for $10 \mathrm{~min}$. Respective supernatant was added to the 96-well strip coated with MMP- 2 antibody or MMP-9 antibody and incubated at $4^{\circ} \mathrm{C}$ overnight. After washing with wash buffer 3 times, $50 \mu$ lassay buffer was added into the well, followed by adding $50 \mu \mathrm{l}$ detection reagent. After incubation at $37^{\circ} \mathrm{C}$ for $1 \mathrm{~h}$, OD405 was measured with microplate reader (BioTek Instruments, Inc., Winooski, VT, USA) (19).

NSCLC xenograft nude mouse model. To test the oncogenic phenotypes of miR-768-3p in NSCLC, we established NSCLC xenograft nude mouse model. Forty-five nude mice (BALB/c strain, 4-5 weeks old, 18-20 g) were purchased from Beijing HFK Bioscience Co., Ltd. (Beijing, China). Mice were housed and raised in the laboratory animal center of the Affiliated Cancer Hospital of Kunming Medical University. Animal use and treatment was approved by the Animal Ethics Committee of Kunming Medical University. Mice were randomly assigned to each of the following 9 groups $(n=5)$ : normal BEAS-2Btreated group; miR-768-3p overexpression human NSCLC cell (A549/HCC4006-miR-768-3p-mimics)-treated groups; miR-768-3p functional deficient NSCLC cell (A549/HCC4006miR-768-3p-antagomir)-treated groups; matched control groups (A549/HCC4006-mimics-NC and A549/HCC4006antagomir-NC). As described before, cells were harvested, digested and injected intradermally into the left axilla of the

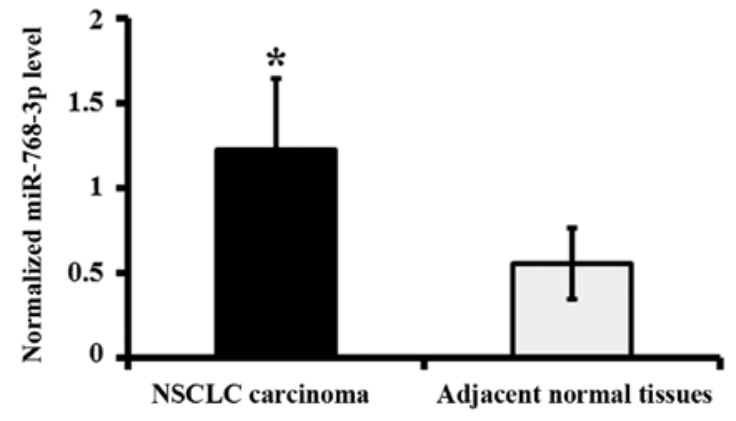

Figure 1. Abnormal expression of miR-768-3p in NSCLC cases. The expression level of miR-768-3p in human NSCLC tissues and the matched adjacent tissues normalized by U6 were determined by qRT-PCR $(n=83)$. Data are represented as mean $\pm \mathrm{SD}$. ${ }^{*} \mathrm{P}<0.05$ vs. adjacent normal tissue. NSCLC, nonsmall cell lung cancer.

nude mice $(20,21)$. After seeding, liquid absorption at the injection site, tumor growth (volume and weight), and mouse survival were measured. Tumor volume was measured on days $3,5,9,13,17,21,25$ and 29 post-injection. The largest (a) and smallest diameters (b) of each tumor were measured twice on days $3,5,9,13,17,21,25$ and 29 to estimate tumor volume $(\mathrm{V})$ using the formula: $\mathrm{V}=0.52 \mathrm{xa}^{2} \times \mathrm{b}(20,21)$. On day 29 , all mice were sacrificed for tumor isolation. Then the tumor weight and volume were evaluated. Mean tumor volumes were used to plot tumor growth curves for each group of mice.

Statistical analysis. Data are presented as means \pm standard deviation (SD). The unpaired t-test was used for comparison between groups. Multivariate logistic regression analysis was performed to evaluate the association between miR-768-3p expressional level (low or high), along with clinicopathological characteristics of NSCLC including sex, age, smoking status, pathological type, cell grading (well, moderate or poor differentiation), TNM stage, as well as tumor size. The strength of association was measured using ORs with $95 \%$ CI. Logistic regression was used for ordinal data to estimate adjusted ORs. The cofactors included in regression analysis were sex, age, smoking status, along with various clinicopathological parameters, including pathological type, cell grading, TNM stage, as well as tumor size. Chi-square test and Fisher's exact test were used to assess the survival curves in NSCLC patients with different miR-768-3p expressional levels. The statistical significance of MTT cell activity, apoptosis factions, invasion and migration, as well as the associated protein levels and MMP2/9 activity among miR-768-3p mimics, antagomir and the matched NC control groups was determined using one-way ANOVA. Significance level was predetermined to be $\mathrm{P} \leq 0.05$ unless otherwise indicated. All analyses were conducted with SPSS version 19.0 software (SPSS, Inc., Chicago, IL, USA).

\section{Results}

Abnormal expression of miR-768-3p associated with NSCLC. The miR-768-3p RNA levels were detected in the tumor tissues of NSCLC patients by qRT-PCR analysis. The data showed that the expression levels of miR-768-3p in NSCLC carcinoma were significantly increased compared to that of adjacent normal tissues $(\mathrm{P}<0.05$; Fig. 1). 
Table I. Analysis of miR-768-3p expression in tumors and clinical characteristics of NSCLC patients.

\begin{tabular}{|c|c|c|c|}
\hline & \multicolumn{3}{|c|}{ miR-768-3p expression $(n=83)$} \\
\hline & $\begin{array}{c}\text { Low } \\
\text { level } \\
\mathrm{n},(\%)\end{array}$ & $\begin{array}{c}\text { High } \\
\text { level } \\
\mathrm{n},(\%)\end{array}$ & $\begin{array}{l}\text { Adjusted OR } \\
(95 \% \mathrm{CI})\end{array}$ \\
\hline Sex & 18 & 65 & \\
\hline Male & $8(9.6)$ & $45(54.2)$ & $36.89(20.35-46.85)$ \\
\hline Female & $10(12)$ & $20(24.2)$ & $3.18(1.23-5.62)$ \\
\hline \multicolumn{4}{|l|}{ Age (years) } \\
\hline$<50$ & $8(9.6)$ & $7(8.4)$ & $1.68(0.68-2.98)$ \\
\hline $50-65$ & $5(6)$ & $54(65.2)$ & $46.98(29.66-58.47)$ \\
\hline$>65$ & $5(6)$ & $4(4.8)$ & $1.36(0.54-3.08)$ \\
\hline \multicolumn{4}{|c|}{ Smoking status } \\
\hline Never & $10(12)$ & $18(21.8)$ & $9.15(3.64-15.22)$ \\
\hline Ever & $8(9.6)$ & $47(56.6)$ & $39.88(29.87-46.89)$ \\
\hline \multicolumn{4}{|c|}{ Pathological type } \\
\hline Adeno- & $3(3.6)$ & $41(49.5)$ & $30.98(21.36-43.68)$ \\
\hline Squamous & $7(8.4)$ & $16(19.3)$ & $13.02(9.65-23.87)$ \\
\hline Large & $8(9.6)$ & $8(9.6)$ & $2.36(1.11-3.82)$ \\
\hline \multicolumn{4}{|c|}{ Differentiation } \\
\hline Poor & $5(6)$ & $17(20.4)$ & $21.88(15.24-31.49)$ \\
\hline Moderate & $6(7.2)$ & $39(47)$ & $39.72(26.81-48.32)$ \\
\hline Well & $7(8.7)$ & $9(10.7)$ & $1.58(0.54-2.49)$ \\
\hline \multicolumn{4}{|l|}{ TNM stage } \\
\hline I & $6(7.2)$ & $25(30.1)$ & $24.33(18.62-36.27)$ \\
\hline II & $7(8.7)$ & $26(31.3)$ & $25.89(17.12-32.66)$ \\
\hline III & $5(6)$ & $14(16.7)$ & $10.33(6.87-15.94)$ \\
\hline \multicolumn{4}{|c|}{ Tumor size (cm) } \\
\hline$\leq 3$ & $7(8.4)$ & $25(38.5)$ & $20.13(12.53-32.83)$ \\
\hline$>3$ & $8(9.6)$ & $40(61.5)$ & $44.62(31.35-57.24)$ \\
\hline
\end{tabular}

CI, confidence interval; OR, odds ratio. Values in bold, $\mathrm{P}<0.05$.

According to the miR-768-3p expression level, we divided all the enrolled patients into two groups: Those with less than or equal to median of miR-768-3p expression levels (low level) and those with more than median of miR-768-3p expression levels (high level). The median was used as cut-off (22).

The association between miR-768-3p status and various clinicopathological characteristics results determined by ORs revealed a positive trend for miR-768-3p level and sex (male, $\mathrm{P}=0.0009)$, age $(50-65, \mathrm{P}=0.00012)$, smoking status (ever, $\mathrm{P}=0.0004)$, pathological type (adenocarcinomas), cell grading (moderate, $\mathrm{P}=0.00051$; poor, $\mathrm{P}=0.002)$, $\mathrm{TNM}$ stage (I, $\mathrm{P}=0.001$; II, $\mathrm{P}=0.001)$, as well as tumor size $(>3, \mathrm{P}=0.0002$; Table I).

miR-768-3p is associated with survival rate of NSCLC. The 60 months (5-year) survival rate of TNM stage 1 is $>94 \%$, while it is $<10 \%$ in patients with TNM stage II-III. Within a period of 60 months of the follow-up, 13 NSCLC related deaths occurred. All of the deaths come from patients with

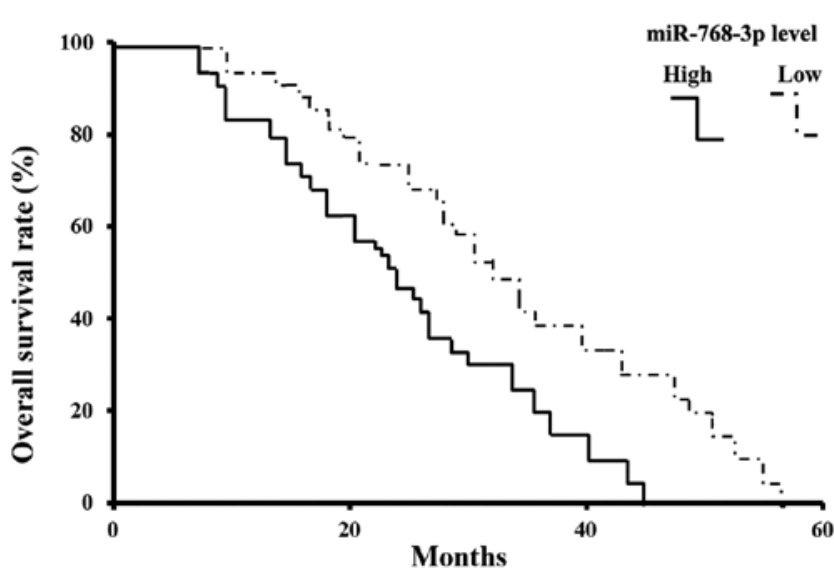

Figure 2. Survival curves of NSCLC patients with miR-768-3p level. NSCLC patients with high miR-768-3p expression had significantly worse total survival than those with low expression. Overall survival rate in patients with high miR-768-3p expression level (miR-768-3p >median) was markedly higher than those with low miR-768-3p expression level (miR-768-3p $\leq$ median)

miR-768-3p positive tumors. Kaplan-Meier estimated the overall survival rate based on tissue miR-768-3p expression in the patients with a follow-up period of 5 years (Fig. 2). In the entire cohort, the overall survival rate of patients with miR-768-3p low expressed tumors were significantly higher than that of those with miR-768-3p positive tumors (83.83 vs. 17.24\%; log-rank test: $\left.\chi^{2}=22.96, \mathrm{P}=0.000002, \mathrm{P}<0.05\right)$ (Fig. 2).

The expression of miR-768-3p in NSCLC cell lines. The level of miR-768-3p was detected in BEAS-2B, A549 and HCC4006 human NSCLC cell lines by qRT-PCR. BEAS-2B served as normal control.

The data showed that miR-768-3p increased in either A549 or HCC4006 human NSCLC cell lines, compared with that of BEAS-2B cell line ( $\mathrm{P}<0.05$; Fig. 3A), respectively. After transduction with miR-768-3p mimics, the expression of miR-768-3p was significantly upregulated in BEAS-2B, A549 and HCC4006 human NSCLC cell lines than that of the NC treated group, respectively $(\mathrm{P}<0.05$; Fig. 3B).

Reversely, the expression of miR-768-3p was significantly downregulated following transduction with miR-768-3p antagomir in the BEAS-2B, A549 and HCC4006 NSCLC cell lines, compared with that of the matched $\mathrm{NC}$ treated group, respectively $(\mathrm{P}<0.05$; Fig. $3 \mathrm{C})$.

Effects of miR-768-3p on the proliferation of NSCLC cell lines. We assessed the effect of miR-768-3p overexpression or knockdown on the regulation of NSCLC cells viability. Treatment with miR-768-3p-mimics significantly increased the cell proliferation in either of A549 cells or HCC4006 cells, compared with that of NC-treated group, respectively. MTT assay also showed that miR-768-3p knockdown by miR-768-3p antagomir transduction caused significantly decrease in cell viability of either A549 or HCC4006 cell lines. However, the effects of neither miR-768-3p mimics nor antagomir transduction were confirmed in BEAS-2B cells (vs. NC treated control, P>0.05; Fig. 4).

Alterations of apoptosis in NSCLC cells after miR-768-3p regulation. The apoptosis rate of NSCLC after miR-768-3p 

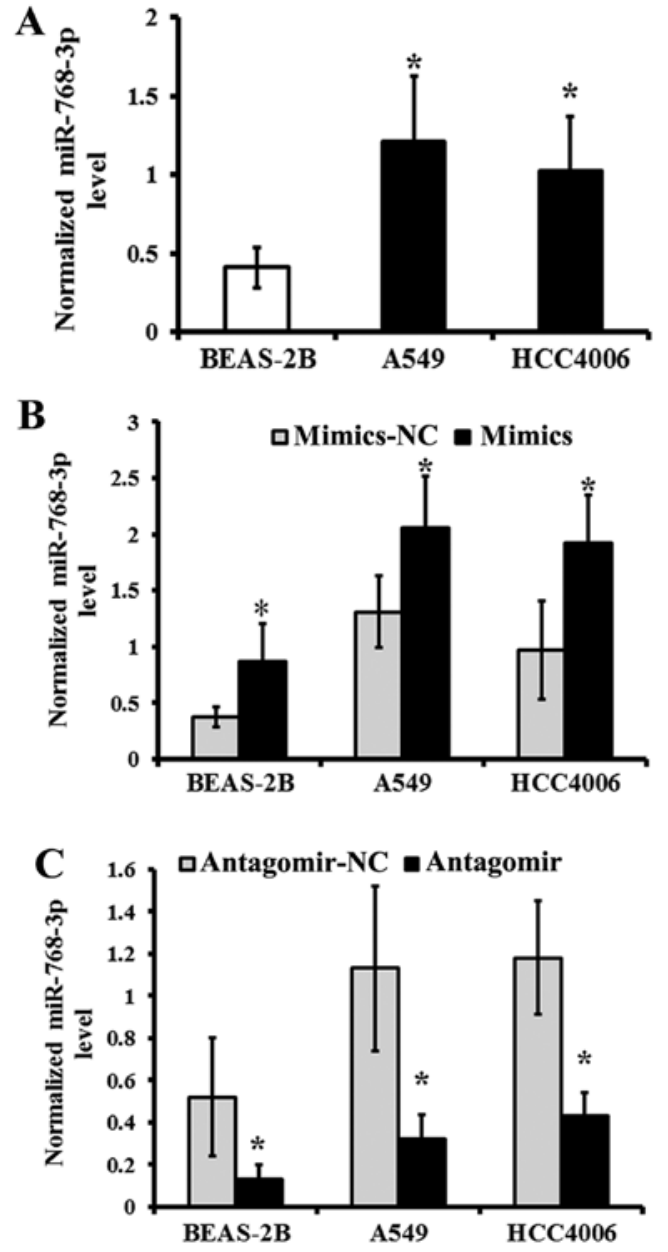

Figure 3. Detection of miR-768-3p level in NSCLC cells. (A) miR-768-3p level increased in human NSCLC cell lines compared with that of BEAS-2B cell line. ${ }^{~} \mathrm{P}<0.05$ vs. BEAS-2B. (B) Transduction with miR-768-3p mimics increased miR-768-3p levels in different cell lines, compared with the matched mimics-NC control. " $\mathrm{P}<0.05$ vs. mimics-NC control. (C) miR-768-3p expression was knocked down by transfecting NSCLC cells treated with miR-768-3p antagomir, when compared with an antagomir-NC transfected cells. ${ }^{*} \mathrm{P}<0.05$ vs. antagomir-NC control. The data represent the means $\pm \mathrm{SD}$ $(n=5)$.

mimics/antagomir transduction was determined by FCM. There were less apoptotic NSCLC cells in A549 and HCC4006 groups treated with miR-768-3p mimics, when compared with that of the matched $\mathrm{NC}$ treated groups, respectively ( $\mathrm{P}<0.05$; Fig. 5A and B). The data also showed that there was a significant increase in the apoptosis rate in miR-768-3p antagomir infected cells relative to the matched control NC infected ones, respectively ( $\mathrm{P}<0.05$; Fig. 5Cand D). However, there was no significant different in the apoptosis of human normal BEAS-2B cells ( $\mathrm{P}>0.05$; Fig. 5A-D).

The expression of apoptosis associated proteins Fas and FasL were also determined in NSCLC cells after miR-768-3p mimics/antagomir treatment. Consistence with the apoptosis assay, compared with the matched NC control, miR-768-3p mimics induced increase of FasL and decrease of Fas expressions, while miR-768-3p antagomir decreased FasL and increased Fas levels, respectively (Fig. 5E and F).

Effect of miR-768-3p on migration, invasion and MMP activity in NSCLC cells. Following miR-768-3p regulation,
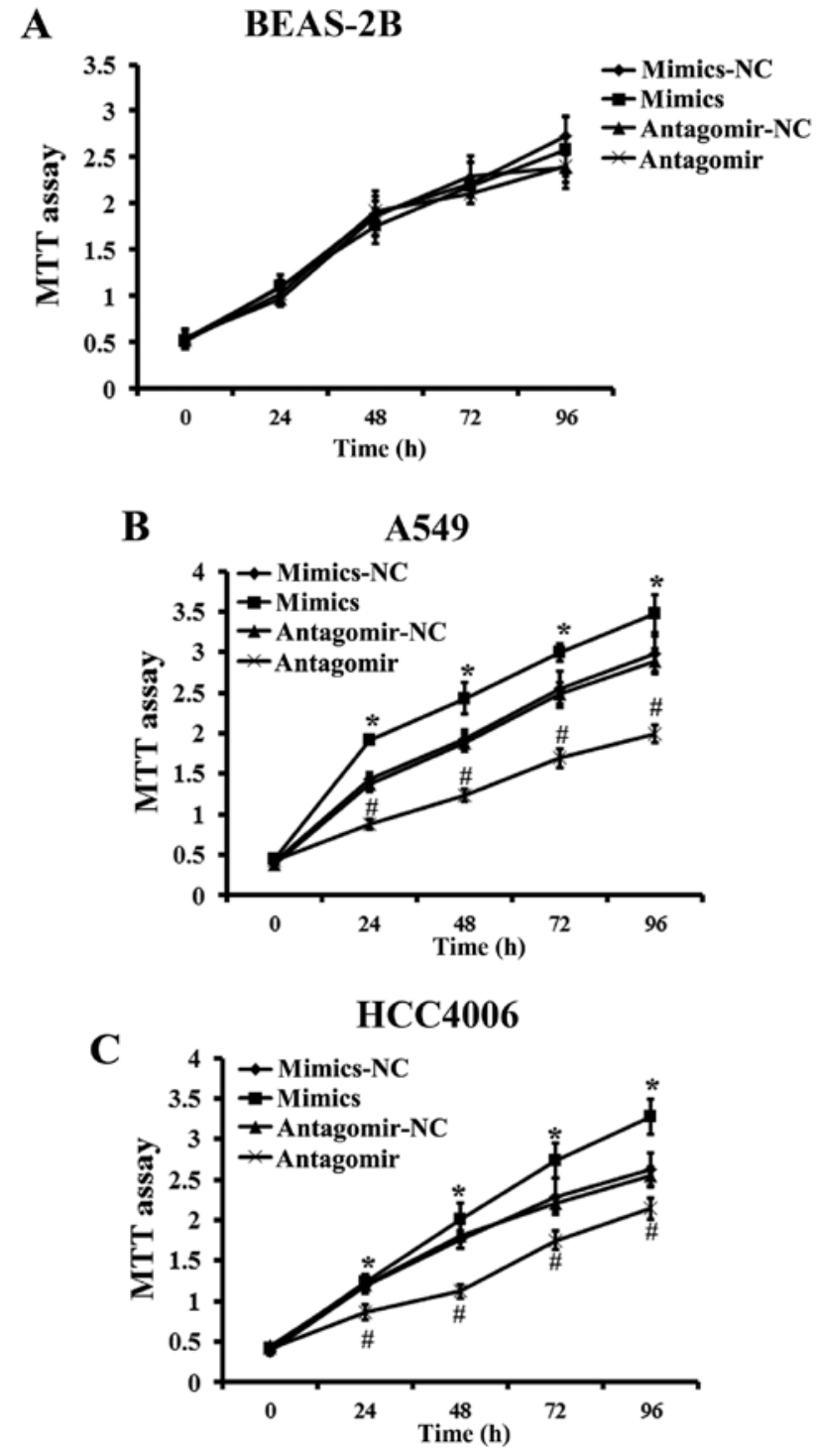

Figure 4 miR-768-3p transfection promoted proliferation of NSCLC cell lines. MTT assay time-course for miR-768-3p mimics/antagomir treated BEAS-2B, A549 and HCC4006. Treatment with miR-768-3p mimics induced significant promotion in proliferation in either A549 (B) or HCC4006 (C) cells, but not in BEAS-2B (A) cells. After miR-768-3p downregulation, both A549 and HCC4006 cells showed significant decrease in proliferation compared with that of the matched $\mathrm{NC}$ control ones, respectively $(\mathrm{P}<0.05)$. ${ }^{\text {"P }}<0.05$ vs. mimics-NC control; ${ }^{\prime} \mathrm{P}<0.05$ vs. antagomir-NC control. Values plotted are means $\pm \operatorname{SD}(n=5)$.

we compared the migration and invasion of NSCLC cells following miR-768-3p mimics, miR-768-3p antagomir, as well as the matched control NC transfection.

Scratch wound assay showed a faster migration of the miR-768-3p mimics transfected NSCLC cells than the control mimics-NC transfected ones (Fig. 6A and B). There were significant reductions of crystal violet stained NSCLC cells following miR-768-3p antagomir transfection, in comparison with that of the control NC treated cells, respectively $(\mathrm{P}<0.05$; Fig. 6C). Consistently, MMP-2 and MMP-9 activities were decreased in both A549 and HCC4006 cells following miR-768-3p antagomir transduction (Fig. 6D).

Reversely, transfection with miR-768-3p mimics significantly increased the migration, invasion and the MMP-2/9 activities in 
A

BEAS-2B
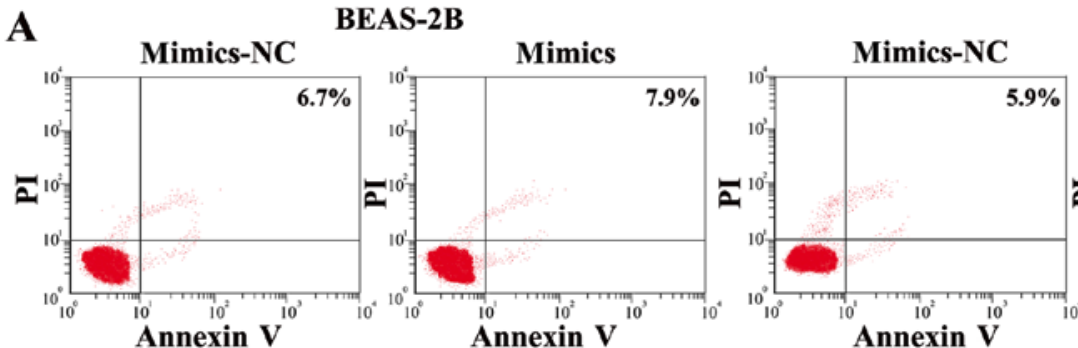

A549

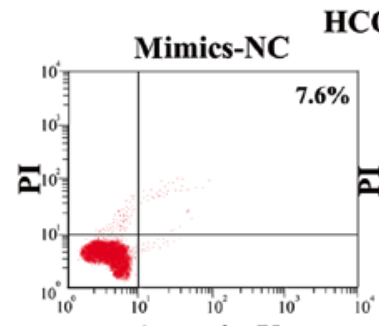

HCC4006

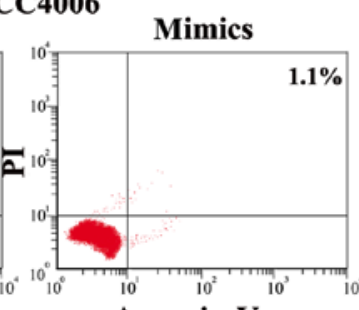

Annexin V

Annexin V

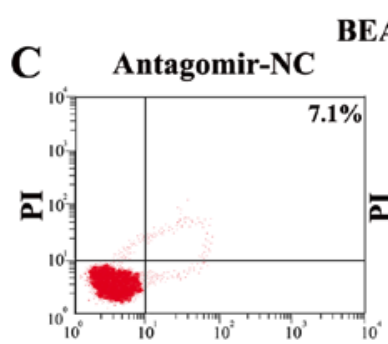

BEAS-2B

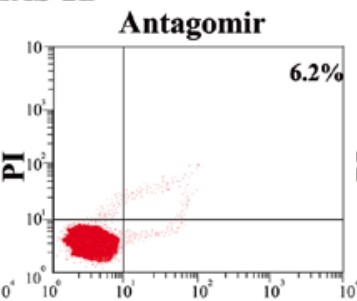

Annexin V

Annexin V

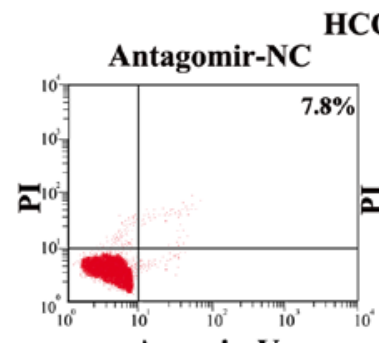

HCC4006
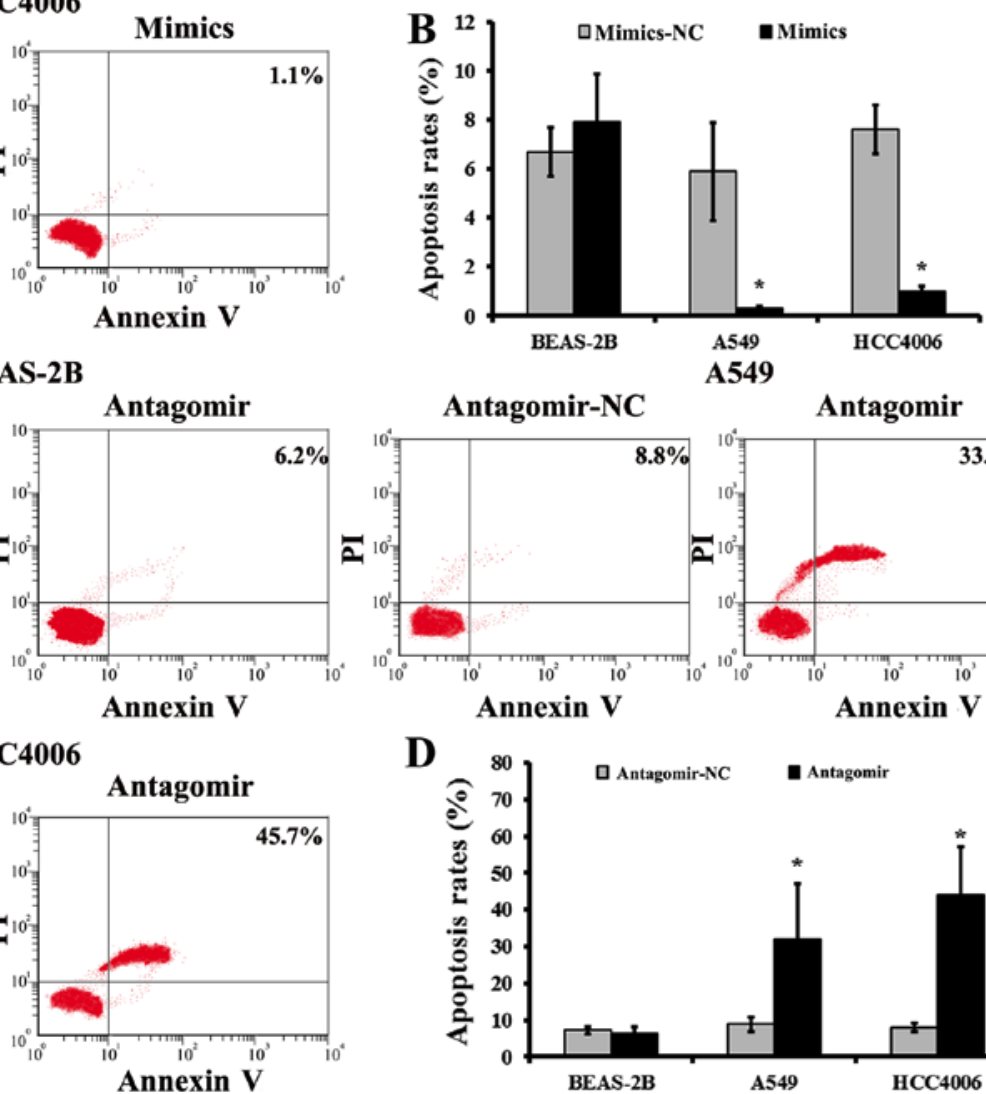

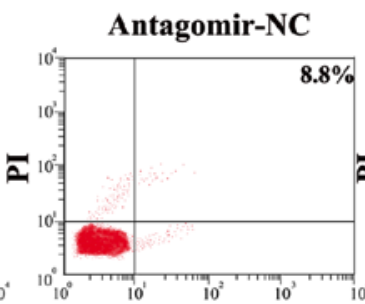

Annexin V

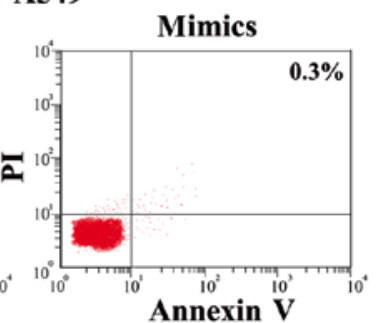

A549

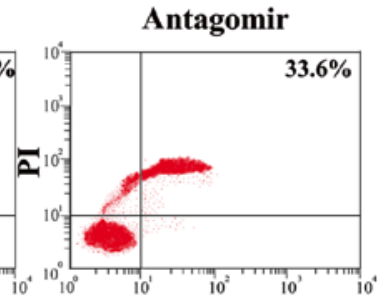

D

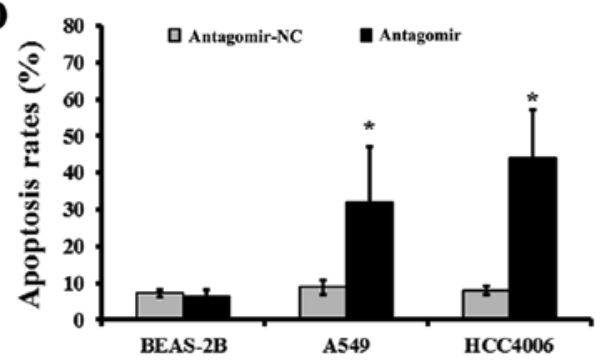

Fas

FasL

E

GAPDH
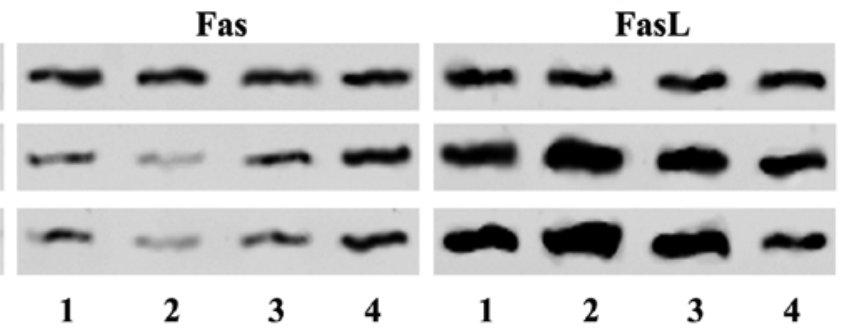

BEAS-2B

A549

HCC4006

F
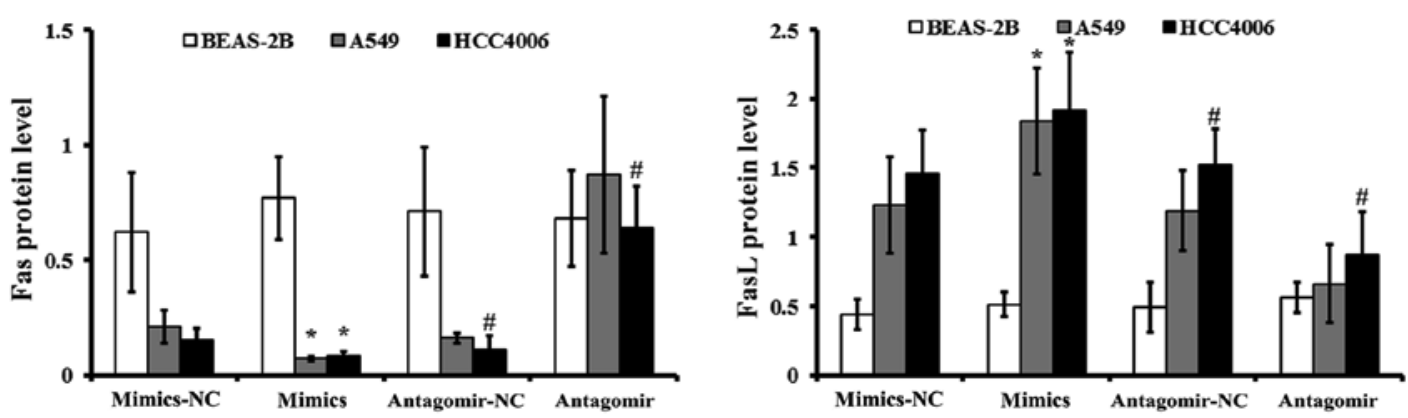

Figure 5. miR-768-3p induces apoptosis of NSCLC cells. (A and C) The effects of miR-768-3p mimics/antagomir on the apoptosis of BEAS-2B, A549 and HCC4006 cells were detected by flow cytometry. (B and D) Quantitative analysis of apoptosis. Quantification showed that the percentage of apoptotic cells in miR-768-3p antagomir transfected groups are significantly higher compared with the percentage in the control NC groups. (E) Expressions of Fas and FasL proteins were determined by western blot analysis in NSCLC cells after different reagent treatments. Lane 1, miR-768-3p mimics-NC; lane 2, miR-768-3p mimics; lane 3, miR-768-3p antagomir-NC; lane 4, miR-768-3p antagomir. (F) Quantitative analysis of Fas and FasL proteins in different cells. ${ }^{*} \mathrm{P}<0.05$ vs. mimics-NC treated cells; ${ }^{*} \mathrm{P}<0.05$ vs. antagomir-NC treated cells. Values plotted are means $\pm \mathrm{SD}(\mathrm{n}=5)$. 
A
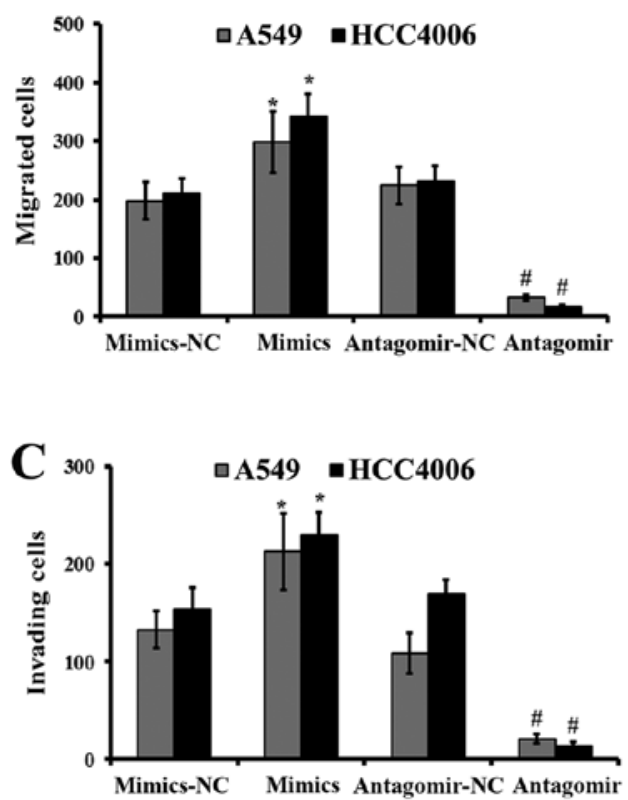

D

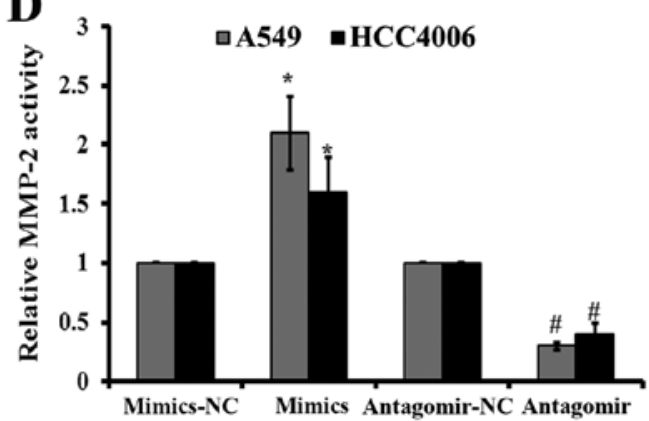

B

$\mathbf{0 h}$

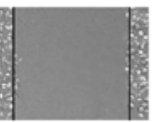

$48 \mathrm{~h}$
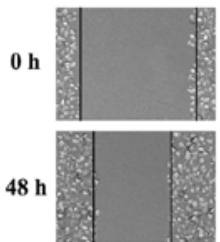

Mimics-NC
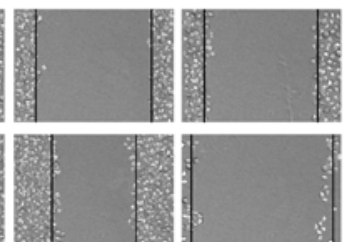

A549
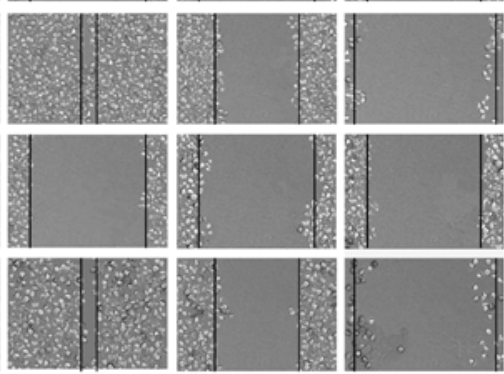

Antagomir

\section{HCC4006}

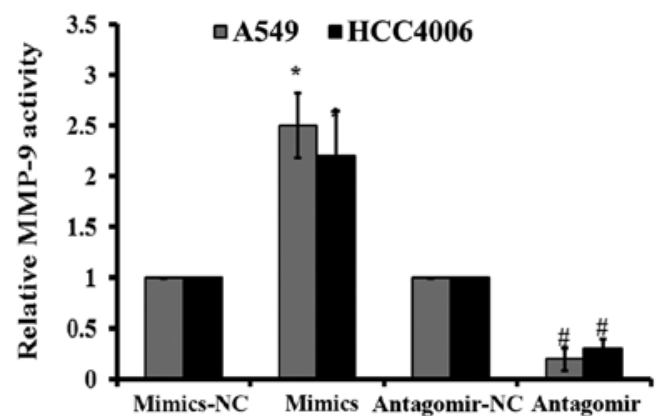

Figure 6. Effect of miR-768-3p on NSCLC cell migration and invasion. (A and B) Scratch wound assay. (A) Number of migrated cells transfected with different treatments; (B) light microscopy images are shown immediately after scratching of the monolayer and $48 \mathrm{~h}$ later. Cells moved more slowly over the wound after miR-768-3p antagomir transduction. (C) Number of invasion cells transfected with different reagents. The Transwell assays indicated that the miR-768-3p mimics promoted A549 and HCC4006 cells passed through the membrane and invaded the bottom chamber, compared with that of NC treated ones. (D) Quantitative analysis of MMP-2/9 activity in miR-768-3p mimics/antagomir transfected A549 and HCC4006 cells. *P<0.05 vs. mimics-NC treated cells; ${ }^{\#} \mathrm{P}<0.05$, vs. antagomir-NC treated cells. Values plotted are means $\pm \mathrm{SD}(\mathrm{n}=5)$.

both kinds of NSCLC cells, compared with that of the matched mimics-NC transduced ones, respectively (P<0.05; Fig. 6).

Effects of miR-768-3p on tumor formation and growth. Tumor formation and growth were measured in mice after injections of different NSCLC cells. Tumor growth was fastest and neoplasms were larger in mice treated with miR-768-3pmimics-treated NSCLC cells compared with the other groups 13 days post-injection ( $\mathrm{P}<0.05$; Fig. 7). Reversely, xenograft tumor formed last and grew slowest in those injected with miR-768-3p-antagomir transfected cells. Tumor sizes were also smaller in the miR-768-3p deficient group than in the other groups between 13 and 29 days post-injection $(\mathrm{P}<0.05)$. These results indicated that $\mathrm{miR}-768-3 \mathrm{p}$ promoted proliferation and tumor formation of NSCLC xenografts in the mouse model.

\section{Discussion}

The present study revealed that: i) miR-768-3p significantly increased in tissues from NSCLC cases, compared with that of adjacent normal control; ii) expression of miR-768-3p in NSCLC tumor cells were associated with clinicopathological factors of NSCLC, including tumor size, histological grade, lymph node metastasis, as well as 5-year survival rate. The data also showed that inhibiting miR-768-3p function by miR-768-3p-atagomir infection caused significant increase in apoptosis and Fas levels, decrease in FasL expression, cell viability, migration and invasion, MMP-2 and MMP-9 activities of A549 and HCC4006 cells, respectively. Moreover, miR-768-3p promoted proliferation and tumor formation in nude mice bearing NSCLC xenograft tumor. These results suggested that abnormal elevated miR-768-3p in NSCLC tumor and cell lines played important role in NSCLC carcinogenic progression, including apoptosis, migration and invasion. The targeting of miR-768-3p may be a potential therapeutic strategy for the treatment of NSCLC.

Aberrant miRNA expression profiles are frequently observed in cancers $(23,24)$ and many miRNAs are implicated in the initiation and progression of cancer and represent potential targets for anticancer treatment $(25,26)$. However, the possible role of miR-768-3p in NSCLC is still not known. Evidence has 

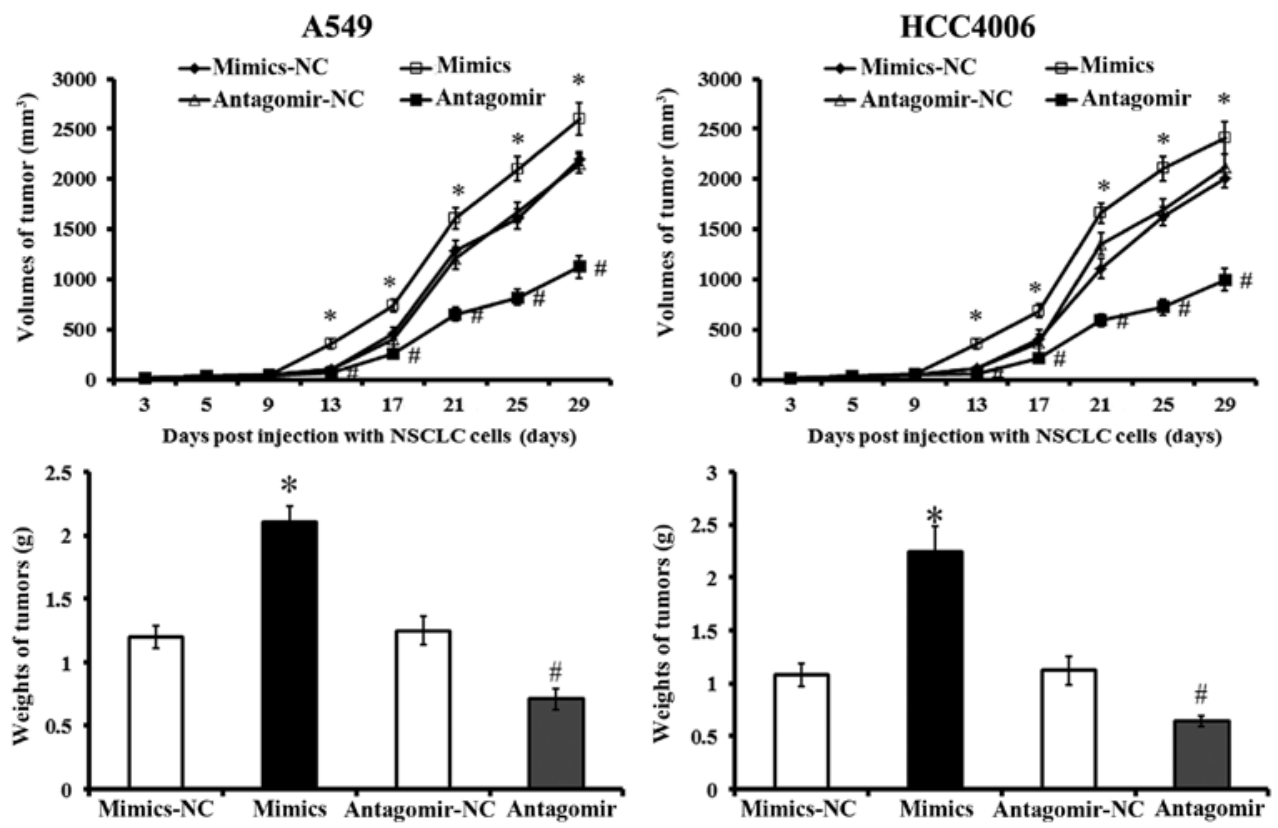

Figure 7. Tumor formation and growth in nude mice after NSCLC xenografts. BEAS-2B, A549 and HCC4006 cells transfected with miR-768-3p mimics, mimics-NC, antagomir or antagomir-NC were injected into nude mice. Tumor size was measured on days 3, 5, 9, 13, 17, 21, 25 and 29 post-injection, respectively. Tumor volume growth curve was plotted. Tumor weights were measured in different groups. $\mathrm{P}<0.05$ vs. mimics-NC treated cells; ${ }^{\text {"}} \mathrm{P}<0.05$ vs. antagomir-NC treated cells. Values plotted are means $\pm \mathrm{SD}(\mathrm{n}=5)$.

shown that abnormal expression of miR-768-3p was observed in various cancers, indicating its oncogenic features. It was described that miR-768-3p were overexpressed in plasma of patients with hepatocellular carcinoma (27) and downregulated in gastric and thyroid tumors $(28,29)$, respectively. Jian and his colleagues (30) reported that in human melanoma downregulation of miR-768-3p as a result of activation of the mitogen-activated protein kinase kinase (MEK)/extracellular signal-regulated kinase (ERK) pathway has an important role in the upregulation of eIF4E and enhancement in protein synthesis. We found that significantly increased miR-768-3p in NSCLC cases were associated with clinicopathological factors of NSCLC. The following detection also revealed the elevated miR-768-3p in NSCLC cells. The data suggested the oncogenic roles of miR-768-3p involved in pathological events of NSCLC.

The data revealed that miR-768-3p inhibition caused significantly decrease in cell viability and proliferation. Induction of apoptosis highly affects cell proliferation. In present study, flow cytometry with Annexin V/PI staining also showed that miR-768-3p antagomir transduction induced apoptosis in NSCLC cells, which was supported by increased expressions of Fas and decreased expressions of FasL. Reversely, miR-768-3p mimics significantly inhibited apoptosis and promoted FasL generation in NSCLC cells. The Fas ligand, FasL played a key role in the initiation of apoptotic pathway (31), and was shown that alterations in Fas/FasL pathway within tumor cells could result in escape from apoptosis and immune surveillance. In malignant cells, their reduced capability to undergo apoptosis in response to some physiological stimuli resulting in a significant survival advantage and possibly contributing to tumorigenesis $(32,33)$. Recent evidence revealed that: i) the Fas-negative NSCLC patients showed significantly lower survival rate than those with Fas-positive ones; ii) FasL protein was increased in most
NSCLC cases (up to 89\%) compared to normal lungs (34). Data demonstrated the reduced membranous Fas expression as a mechanism of apoptotic resistance was considered to play an important part of the pulmonary carcinogenesis. Increased FasL expression is thought to be a basis for the immune evasion in NSCLCs (34). Combined with the above, the present study suggested that miR-768-3p played a role in the apoptotic resistance of NSCLCs by Fas/FasL regulation.

Our results also suggested that abnormally elevated miR-768-3p in NSCLC tumor and cell lines played an important role in NSCLC carcinogenic progression, including migration and invasion. NSCLC has a very poor prognosis and is often characterized by aggressive local invasion, early metastasis and poor response to chemotherapy (35). Consequently, targeting and prevention of cancer cell metastasis is among the biggest hurdles in clinical oncology (36). Although a variety of metastasis-promoting genes have been recently identified to be related to the metastasis of NSCLC, the molecular mechanisms governing this metastasis process are still not completely understood and the treatment efficiency of metastatic NSCLC has not been significantly improved $(37,38)$. During metastasis, cancer cells rely heavily on cell-extracellular matrix (ECM) interactions, cytoskeleton remodeling and gene transcription (39). Taking into account that MMPs such as MMP-2 and MMP-9 can be involved in the development of several human malignancies, as degradation of collagen IV in basement membrane and extracellular matrix facilitates tumor progression, including invasion, metastasis, and angiogenesis, we analyzed their activity (39). The data also revealed that inhibiting miR-768-3p function leads to increased migration and invasion rate, as well as MMP-2/9 activities in NSCLC cells, which indicated that miR-768-3p was involved in metastatic process of NSCLC by promoting NSCLC cells to migrate and invade. 
In conclusion, the results demonstrated that abnormal elevated miR-768-3p in both carcinoma tissues in NSCLC cases and NSCLC cell lines, were associated with clinic pathological factors of NSCLC. Further inhibition of miR-768-3p by miR-768-3p antagomir transduction: i) significantly increased apoptosis by Fas/FasL regulation; ii) promoted proliferation of NSCLC both in vitro and in vivo; iii) increased migration and invasion of NSCLC cells. Just as we expected, transfection with miR-768-3p mimics reversed the effects described above. Our data suggested that miR-768-3p played a role in the apoptotic resistance of NSCLCs by Fas/FasL regulation, and it was involved in metastatic process of NSCLC by promoting NSCLC cells to proliferate, migrate and invade. The targeting of miR-768-3p may be a potential therapeutic strategy for the future treatment of NSCLC.

\section{References}

1. Jemal A, Thun MJ, Ries LA, Howe HL, Weir HK, Center MM, Ward E, Wu XC, Eheman C, Anderson R, et al: Annual report to the nation on the status of cancer, 1975-2005, featuring trends in lung cancer, tobacco use, and tobacco control. J Natl Cancer Inst 100: 1672-1694, 2008.

2. Jemal A, Bray F, Center MM, Ferlay J, Ward E and Forman D: Global cancer statistics. CA Cancer J Clin 61: 69-90, 2011.

3. Torre LA, Bray F, Siegel RL, Ferlay J, Lortet-Tieulent J and Jemal A: Global cancer statistics, 2012. CA Cancer J Clin 65 $87-108,2015$.

4. Chen Z, Fillmore CM, Hammerman PS, Kim CF and Wong KK Non-small-cell lung cancers: A heterogeneous set of diseases. Nat Rev Cancer 14: 535-546, 2014.

5. Herbst RS, Heymach JV and Lippman SM: Lung cancer. N Engl J Med 359: 1367-1380, 2008

6. Wightman B, Ha I and Ruvkun G: Posttranscriptional regulation of the heterochronic gene lin-14 by lin- 4 mediates temporal pattern formation in C. elegans. Cell 75: 855-862, 1993.

7. Hutvágner G and Zamore PD: RNAi: Nature abhors a doublestrand. Curr Opin Genet Dev 12: 225-232, 2002.

8. Ketting RF, Fischer SE, Bernstein E, Sijen T, Hannon GJ and Plasterk RH: Dicer functions in RNA interference and in synthesis of small RNA involved in developmental timing in C. elegans. Genes Dev 15: 2654-2659, 2001.

9. He L, Thomson JM, Hemann MT, Hernando-Monge E, Mu D, Goodson S, Powers S, Cordon-Cardo C, Lowe SW, Hannon GJ, et al: A microRNA polycistron as a potential human oncogene. Nature 435: 828-833, 2005.

10. Zhang WC, Liu J, Xu X and Wang G: The role of microRNAs in lung cancer progression. Med Oncol 30: 675-683, 2013.

11. Saito M, Schetter AJ, Mollerup S, Kohno T, Skaug V, Bowman ED, Mathé EA, Takenoshita S, Yokota J, Haugen A, et al: The association of microRNA expression with prognosis and progression in early-stage, non-small cell lung adenocarcinoma: A retrospective analysis of three cohorts. Clin Cancer Res 17: 1875-1882, 2011.

12. Malleter M, Jacquot C, Rousseau B, Tomasoni C, Juge M, Pineau A, Sakanian V and Roussakis C: miRNAs, a potential target in the treatment of non-small-cell lung carcinomas. Gene 506: 355-359, 2012.

13. Raponi M, Dossey L, Jatkoe T, Wu X, Chen G, Fan H and Beer DG: MicroRNA classifiers for predicting prognosis of squamous cell lung cancer. Cancer Res 69: 5776-5783, 2009.

14. Lin PY, Yu SL and Yang PC: MicroRNA in lung cancer. Br J Cancer 103: 1144-1148, 2010

15. Koeck S, Amann A, Huber JM, Gamerith G, Hilbe W and Zwierzina $\mathrm{H}$ : The impact of metformin and salinomycin on transforming growth factor $\beta$-induced epithelial-to-mesenchymal transition in non-small cell lung cancer cell lines. Oncol Lett 11: 2946-2952, 2016.

16. Hou FQ, Lei XF, Yao JL, Wang YJ and Zhang W: Tetraspanin 1 is involved in survival, proliferation and carcinogenesis of pancreatic cancer. Oncol Rep 34: 3068-3076, 2015.

17. He Z, Huang C, Lin G and Ye Y: siRNA-induced TRAF6 knockdown promotes the apoptosis and inhibits the invasion of human lung cancer SPC-A1 cells. Oncol Rep 35: 1933-1940, 2016.
18. Ouchi Y, Banno Y, Shimizu Y, Ando S, Hasegawa H, Adachi K and Iwamoto T: Reduced adult hippocampal neurogenesis and working memory deficits in the Dgcr8-deficient mouse model of 22q11.2 deletion-associated schizophrenia can be rescued by IGF2. J Neurosci 33: 9408-9419, 2013.

19. Santiago-Gómez A, Barrasa JI, Olmo N, Lecona E, Burghardt H, Palacín M, Lizarbe MA and Turnay J: 4F2hc-silencing impairs tumorigenicity of HeLa cells via modulation of galectin-3 and $\beta$-catenin signaling, and MMP-2 expression. Biochim Biophys Acta 1833: 2045-2056, 2013.

20. Hu T, Chang YF, Xiao Z, Mao R, Tong J, Chen B, Liu GC, Hong Y, Chen HL, Kong SY, et al: miR-1 inhibits progression of high-risk papillomavirus-associated human cervical cancer by targeting G6PD. Oncotarget 7: 86103-86116, 2016.

21. Hu T, Zhang C, Tang Q, Su Y, Li B, Chen L, Zhang Z, Cai T, Zhu Y: Variant G6PD levels promote tumor cell proliferation or apoptosis via the STAT3/5 pathway in the human melanoma xenograft mouse model. BMC Cancer 22;13:251, 2013.

22. He Z, Xia Y, Liu B, Qi X, Li Z, Wang J, Chen L and Chen Y: Down-regulation of miR-452 is associated with poor prognosis in the non-small-cell lung cancer. J Thorac Dis 8: 894-900, 2016.

23. Lu J, Getz G, Miska EA, Alvarez-Saavedra E, Lamb J, Peck D, Sweet-Cordero A, Ebert BL, Mak RH, Ferrando AA, et al: MicroRNA expression profiles classify human cancers. Nature 435: 834-838, 2005.

24. Lin S and Gregory RI: MicroRNA biogenesis pathways in cancer. Nat Rev Cancer 15: 321-333, 2015.

25. Kasinski AL and Slack FJ: Epigenetics and genetics. MicroRNAs en route to the clinic: Progress in validating and targeting microRNAs for cancer therapy. Nat Rev Cancer 11: 849-864, 2011.

26. Chen Y, Gao D, Huang L: In vivo delivery of miRNAs for cancer therapy: challenges and strategies. Adv Drug Deliv Rev 81C: 128-141, 2011.

27. Zhou J1, Yu L, Gao X, Hu J, Wang J, Dai Z, Wang JF, Zhang Z, Lu S, Huang X, Wang Z, et al: Plasma microRNA panel to diagnose hepatitis B virus-related hepatocellular carcinoma. J Clin Oncol 29: 4781-4788, 2011.

28. Vriens MR, Weng J, Suh I, Huynh N, Guerrero MA, Shen WT, Duh QY, Clark OH and Kebebew E: MicroRNA expression profiling is a potential diagnostic tool for thyroid cancer. Cancer 118: 3426-3432, 2012.

29. Guo J, Miao Y, Xiao B, Huan R, Jiang Z, Meng D and Wang Y: Differential expression of microRNA species in human gastric cancer versus non-tumorous tissues. J Gastroenterol Hepatol 24: 652-657, 2009

30. Jian Q, An Q, Zhu D, Hui K, Liu Y, Chi S and Li C: MicroRNA 340 is involved in UVB-induced dendrite formation through the regulation of RhoA expression in melanocytes. Mol Cell Biol 34: 3407-3420, 2014

31. Nagata S and Golstein P: The Fas death factor. Science 267 1449-1456, 1995.

32. Wyllie AH, Kerr JF and Currie AR: Cell death: The significance of apoptosis. Int Rev Cytol 68: 251-306, 1980.

33. Thompson CB: Apoptosis in the pathogenesis and treatment of disease. Science 267: 1456-1462, 1995

34. Li Y, Xu KP, Jiang D, Zhao J, Ge JF and Zheng SY: Relationship of Fas, FasL, p53 and bcl-2 expression in human non-small cell lung carcinomas. Int J Clin Exp Pathol 8: 13978-13986, 2015.

35. Forde PM and Ettinger DS: Targeted therapy for non-small-cell lung cancer: Past, present and future. Expert Rev Anticancer Ther 13: 745-758, 2013.

36. Frisch SM, Schaller M and Cieply B: Mechanisms that link the oncogenic epithelial-mesenchymal transition to suppression of anoikis. J Cell Sci 126: 21-29, 2013.

37. Choi YH, Burdick MD, Strieter BA, Mehrad B and Strieter RM: CXCR4, but not CXCR7, discriminates metastatic behavior in non-small cell lung cancer cells. Mol Cancer Res 12: 38-47, 2014.

38. Jin Y, Li F, Zheng C, Wang Y, Fang Z, Guo C, Wang X, Liu H, Deng L, Li C, et al: NEDD9 promotes lung cancer metastasis through epithelial-mesenchymal transition. Int J Cancer 134: 2294-2304, 2014

39. Xu LJ, Wang YC, Lan HW, Li J and Xia T: Grb2-associated binder-2 gene promotes migration of non-small cell lung cancer cells via Akt signaling pathway. Am J Transl Res 8: 1208-1217, 2016. 